\title{
Sociologie d'intervention : historique et fondements
}

Denis Bernardeau Moreau

\section{(2) OpenEdition}

1 Journals

Édition électronique

URL : https://journals.openedition.org/ress/2872

DOI : $10.4000 /$ ress.2872

ISSN : 1663-4446

Éditeur

Librairie Droz

\section{Édition imprimée}

Date de publication : 27 novembre 2014

Pagination : 191-220

ISBN : 978-2-600-01866-1

ISSN : 0048-8046

\section{Référence électronique}

Denis Bernardeau Moreau, "Sociologie d'intervention : historique et fondements », Revue européenne des sciences sociales [En ligne], 52-2 | 2014, mis en ligne le 01 janvier 2018, consulté le 21 septembre 2021. URL : http://journals.openedition.org/ress/2872 ; DOI : https://doi.org/10.4000/ress.2872 


\title{
SOCIOLOGIE D'INTERVENTION: HISTORIQUE ET FONDEMENTS
}

DENIS BERNARDEAU MOREAU

Université Paris-Est Marne-la-Vallée - ACP (EA 3350)

Denis.Bernardeau@univ-mlv.fr

\begin{abstract}
Résumé. Cet article expose les principales caractéristiques de la sociologie d'intervention, depuis ses origines dont on peut situer les prémices dans les travaux monographiques de Frédéric Le Play menés il y a 150 ans, jusqu'à ses formes actuelles. Assez peu évoquée dans la littérature sociologique ou alors au détour d'un paragraphe ou dans des annexes méthodologiques, la sociologie d'intervention, bien que largement pratiquée en dehors du champ académique, manque encore aujourd'hui de lisibilité et de reconnaissance institutionnelle. En proposant une lecture approfondie des principales théories et conceptions développées durant les deux siècles écoulés, cet article se donne pour objectif d'expliciter, à travers le prisme sociologique, les concepts fondamentaux de la sociologie d'intervention.
\end{abstract}

Mots-clés: auto-analyse, demande sociale, empirisme, enquête monographique, Frédéric Le Play, immersion, sociologie d'intervention.

\begin{abstract}
This article states the main characteristics of intervention sociology, from its origins, which can be situated in Frédéric Le Play's monographic work carried out 150 years ago, until its present forms. Rather seldom mentioned in sociological literature or, if so, in the course of a paragraph or in methodological appendixes, intervention sociology, although widely practised outside the academic field, today still lacks legibility and institutional recognition. By proposing a thorough reading of the main theories and conceptions developed during the past two centuries, this article aims at explaining, through the sociological prism, the fundamental concepts of intervention sociology.
\end{abstract}

Keywords: empirism, Frédéric Le Play, immersion, monographic inquiry, self-analysis, social demand, sociology of intervention. 


\section{INTRODUCTION Les postures d'intervention}

Si l'analyse sociologique de l'intervention peut soulever les questions habituelles à tout débat scientifique, expliciter les manières concrètes de conduire cette intervention en situation soulève davantage de réserves et de questionnements. Question de légitimité, l'intervention serait, selon certains, réservée aux experts ${ }^{1}$ et autres professionnels de terrain, laissant aux sociologues le soin de l'interprétation scientifique et distanciée des phénomènes sociaux. Pour les tenants de cette posture², la sociologie doit être pensée davantage à la manière durkheimienne et wébérienne, c'est-à-dire «à partir de son extériorité » (Lapeyronnie, 2004, p. 628) et moins comme le produit d'un dialogue intersubjectif avec les acteurs sociaux. L'intervention serait donc réservée aux praticiens et ne saurait être assimilée à la sociologie, au risque de dissoudre la seconde dans la première. Notre position de sociologue d'intervention ne retient évidemment pas cette dichotomie. Posture scientifique et manière de concevoir le métier de sociologue, la sociologie d'intervention est une science qui ne peut se réduire à «un simple prolongement d'une pratique de recherche, à un sous-produit » (Osty, 200I, p. I68). Notre intention, dans cet article, est d'exposer les principales caractéristiques de cette branche de la sociologie, depuis ses origines dont on peut situer les prémices dans les travaux monographiques de Frédéric Le Play menés il y a 150 ans, jusqu’à sa forme réactualisée aujourd'hui. Assez peu évoquée dans la littérature sociologique française ou alors au détour d’un paragraphe ou dans des annexes méthodologiques - quand

I C'est-à-dire ceux qui, face à une situation problématique qui ne peut être surmontée par l'exercice professionnel habituel, vont mobiliser un savoir de spécialistes qui conduit à un avis rendu aux commanditaires pour les aider dans leur prise de décision (Trépos, 1996, p. 15). Cette figure de l'expert tend à se développer aujourd'hui en raison d'une prégnance accrue des «problématiques de l'urgence» (ibid.) amenant le spécialiste à devoir apporter des réponses immédiates aux sollicitations pressantes émanant de la société.

2 Pour Lapeyronnie, une partie croissante de la sociologie française serait même de plus en plus marquée par une sorte d'«académisme radical». La conséquence de cette posture est «de voir dans la théorie la source d'une vérité objective, alors que l'expérience et la parole des acteurs ne peuvent être conçues que comme des illusions, illusions destinées à être démenties par le «dévoilement sociologique» (2004, p. 624). 
le sociologue l'évoque, c'est la plupart du temps pour préciser une posture empirique ou clinique (Crozier et Friedberg, 1977; Friedberg, 1993) ou pour rendre compte d'une situation d'expertise (Uhalde, 200I ; Felder, 2007) - la sociologie d'intervention, bien que largement pratiquée en dehors du champ académique (Piriou, 2006), manque encore aujourd'hui de lisibilité et de reconnaissance institutionnelle. À ce titre, les ouvrages récents de Gilles Herreros (2000, réédité en 2009, 2008), de Didier Vrancken et Olgierd Kuty (200I³) ou d'Yves Gilbert (2009) constituent des sources scientifiques utiles. Avant eux, Antoine Savoye y avait consacré une thèse en 1979 et Rémi Hess un ouvrage entier paru en 198I. Au regard d'une littérature peu abondante sur le sujet, définir les contours de cette sociologie n’en est que moins aisé. Les courants théoriques les plus divers peuvent s'en réclamer ouvertement ou se retrouver, parfois sans le savoir, sous une même bannière ${ }^{4}$. Mais avant d'aller plus loin sur les questions de concepts et de postures, il convient de revenir sur les moments clés susceptibles de nous éclairer sur les origines de la sociologie d’intervention. Schématiquement, on peut dire avec Savoye et Hess que la sociologie d'intervention puise ses fondements dans trois courants théoriques principaux - la sociologie clinique, la psychosociologie et la microsociologie - certes différents dans leurs concepts mais convergents dans leurs rapports à l'objet. L'idée d'intervention et ses premières formes de théorisation ont été portées notamment par la sociologie clinique américaine des années 1960 puis française trente ans plus tard. Que ce soit Saul Alinsky (1978) avec ses méthodes d’intervention très «intrusives » Danilo Dolci et ses études sur le peuple sicilien (1957),

3 Notons que cet ouvrage juxtapose la sociologie et l'intervention plus qu'il n'en forme un concept unifié.

4 Voir à ce propos la classification extrêmement large et très hétéroclite de Guy Minguet (200I) et la critique qu'en fait notamment Herreros (2009, p. 34-35) regrettant la confusion des classifications entre les sociologies portant sur l'analyse de l'intervention (classification analytique) et les sociologies portant sur les méthodes d'intervention (classification méthodologique).

5 Le sociologue doit chercher à créer de l'agitation et laisser croire aux individus qu'ils ne peuvent s'en sortir seuls. Dans ce jeu un peu pervers, il tente de se rendre indispensable. Et pour cela, tout est permis, de l'agitation auto-suscitée jusqu'au conflit volontairement provoqué, de telle manière que le sociologue apparaisse comme la seule solution possible pour régler le problème: «Un problème est quelque chose sur quoi on a prise [...] Que fait l'organisateur? ॥ transforme en un problème ce qui n'était qu'un mauvais état de choses» (Alinsky, 1978, p. 173). 
Alfred McClung Lee et ses travaux sur l'humanist sociology (1966) ${ }^{6}$ ou encore Paulo Freire (1996 [1964]), et à sa suite Bernard Dumas et Michel Seguier (1999) à l'origine du concept d'«enquête conscientisante »', toutes ces approches appellent de leurs vœux un rapport plus étroit et immédiat entre les chercheurs et leurs objets/sujets dans le but d'agir sur l'organisation et d'œuvrer à son changement. Sur un versant plus psychosociologique, Kurt Lewin et sa méthode de la recherche-action, Elliott Jacques et la socio-analyse, Jacob Levy Moreno et la sociométrie ou encore Carl Rogers et la théorie de la non-directivité font office de précurseurs. En France, ces travaux trouvent un écho important grâce à des chercheurs comme Robert Pagès, Vincent De Gaulejac, George Lapassade ou encore Jean Dubost ${ }^{8}$. Pour les psychosociologues, intervenir signifie agir dans une organisation sociale à la demande de celle-ci et en vue de faciliter son changement (Lapassade, 2006, p. 5 I). Il s'agit d'encourager l'autogestion du groupe et chercher avec lui les solutions à ses problèmes. Le psychosociologue est d'abord celui qui, dans sa pratique, attribue un rôle essentiel à la «parole sociale» (ibid., p. 63). Au fond, toutes ces théories cliniques et psychosociologiques ont un même socle commun propre à toute pratique d'intervention : elles recherchent, par la méthode et une certaine posture empirique, à susciter la prise en charge du groupe par lui-même afin de lui permettre de développer son autonomie et sa capacité à gérer ses conflits.

Le travail du chercheur consiste, dès lors, à partir de ce problème, voire à l'amplifier, pour justifier sa présence dans l'organisation.

6 Dans Multi-valent Man, son ouvrage publié en 1966, Lee distingue le sociologue classique et le sociologue d'intervention dont la participation est directe dans les situations qu'il étudie. Pour lui, certains objets sociaux ne peuvent être approchés autrement que par l'immersion du sociologue.

7 La finalité de cette recherche est d'amener les individus à participer à leur propre processus de «conscientisation». Les groupes participent à l'enquête à chaque étape de son déroulement et détiennent seuls le pouvoir de décider de ses orientations (les points principaux de cette approche sont repris dans l'ouvrage de Colette Humbert et Jean Merlo [1978]).

8 On pourra lire à ce propos la synthèse de ces travaux dans les ouvrages de Dubost (1987 et 2006) et de Lapassade (2006 [1965]). 
En proposant une lecture longitudinale des principales théories et conceptions développées durant les deux siècles écoulés, notre article se donne pour objectif d'expliciter, à travers le prisme sociologique, les concepts fondamentaux de la sociologie d'intervention. Pour cela, nous distinguerons deux parties. La première partie se veut avant tout historique en rappelant chronologiquement les principales sources scientifiques qui ont alimenté les divers courants attachés à la posture d'intervention. Notre intention n'est pas tant de commenter et critiquer les apports théoriques et paradigmatiques considérables des approches scientifiques. Dans notre réflexion sur les fondements historiques de l'intervention sociologique, nous nous intéresserons principalement à une analyse des méthodes et postures revendiquées par ces courants sociologiques majeurs qui donnent corps à l'intervention. Les contours de la posture d'intervention seront ensuite précisés dans la seconde partie de cet article. Il s'agira, en se référant aux différents courants sociologiques qui trouveront écho en France, en convoquant les «sociologues du vécu (Dasseto, 2006, p. 44), d'énoncer les principaux traits caractéristiques de cette sociologie d'intervention. L’une de ses spécificités qui la distingue des autres sociologies est le rapport subjectif qu'elle établit avec l'environnement social (Kaloara et Savoye, 1989). La volonté d’apporter des réponses pratiques à la demande sociale n'exclut toutefois pas, comme nous le verrons dans la conclusion, la propension de la sociologie d'intervention à effectuer des montées en généralité. Si la connaissance que produit l'immersion en situation doit être utile en premier lieu aux individus concernés, elle n’est, précise Dominique Schnapper (20I I, p. 3I), que l'une des données sur lesquelles peut se fonder l'effort d'intelligibilité. Au-delà, il faut pouvoir situer, autant que possible, l'organisation dans un contexte plus global. 


\section{AUX ORIGINES, UNE SOCIOLOGIE MONOGRAPHIQUE ET EMPIRIQUE}

On a trop tendance à considérer, écrivent Vrancken et Kuty (200I, p. I3), que la sociologie d'intervention repose sur trois courants : l'institutionnalisme, l'actionnalisme et l'analyse stratégique. Or, pour comprendre la sociologie d'intervention, il est utile de remonter plus loin dans le temps, de revenir à ses origines, de la mettre en perspective avec les courants dont elle est en partie issue. Nous l'avons vu dans l'introduction, la sociologie d'intervention s'inspire de sources très diverses à la frontière entre sciences sociales et sciences humaines. Du point de vue sociologique, on peut, avec Savoye (1979) et Hess (198I), en situer les prémices dans la microsociologie monographique de Le Play, relayée par les sociologues empiristes américains notamment de l'école de Chicago puis redynamisée en Europe. Toutes ces approches partagent une même préférence pour une lecture davantage microsociologique, en portant leur intérêt sur l'individu sujet et acteur, et en privilégiant l'observation directe et engagée depuis l'intérieur des situations sociales et des groupes restreints. Pour elles, la sociologie ne doit pas s'en tenir à expliquer et à comprendre, elle doit aussi permettre d'agir avec les acteurs.

\section{I.I. LE MICROCOSME FAMILIAL COMME MIROIR DE LA SOCIÉTÉ}

«L'observation directe des faits peut seule, en matière scientifique, conduire à des conclusions rigoureuses et les faire accepter » (Le Play, 2000 [1862], p.203). Si Le Play ${ }^{9}$ peut être considéré comme un précurseur de la sociologie d'intervention, c'est parce qu'il a conduit avec la plus grande rigueur méthodologique de multiples enquêtes monographiques. À une époque où la sociologie se bâtit à partir de la philosophie sociale - le positivisme comtien s'affirme en Europe tandis que la légitimation universitaire de la sociologie par Durkheim reste encore à faire - cet auteur marque une rupture en multipliant les études microsociales. «Je n’ai pas tardé [...] à reconnaître l'insuffisance des vues théoriques», écrit-il en I848, «et je dois presque exclusivement à mes 
relations avec les ouvriers les connaissances plus exactes qu'il ma été permis d’acquérir » (I848, p. I2). Plus tard, il ajoute: «Pour acquérir la connaissance approfondie des phénomènes spéciaux à chaque genre d'ateliers, j’ai dû souvent rester pendant des jours et des nuits au contact des ouvriers » (I88I, p. 5-6). Ses enquêtes monographiques portent sur l'organisation sociale des mineurs du Haut-Harz d’Allemagne du Nord (1829), sur les charpentiers de Paris (1856), sur les couvreurs et vitriers de Savoie (1857) ou encore sur les boulangers parisiens (I859). Il publie en I855 un ouvrage largement plébiscité sur les « ouvriers européens » et instille l'idée progressiste d’une réflexion sociale de grande envergure sur le monde du travail ouvrier dans l'ère industrielle. Armé de ses habituels outils d'enquêtes (grilles d'observation, formulaires de comptages et d'inventaires, entretiens et récits de vie), Le Play devient maître dans l'usage de l'observation directe. Pour bien constater les faits sociaux contenus dans le cadre d'une monographie, écrit-il, il faut remplir deux conditions: l'adhésion et la confiance. Une famille se prête volontiers à l'observation et à une enquête minutieuse «si elle s'aperçoit que l'observateur ne cherche à connaître la condition des classes ouvrières que pour établir par des faits les principes qui permettent de l'améliorer » (2000 [1862], p. I5). Contrairement à d’autres chercheurs de son temps plus distants tels Louis-René Villermé, Le Play trouve du plaisir à se mêler à la vie ouvrière avec laquelle il partage chaque instant. Son hypothèse, précise Savoye (198I, p. 316-317), est de prétendre que l'état d'une société peut se soumettre à l'analyse à partir de l'étude directe d'une unité microsociale à condition que celle-ci soit bien choisie (la famille ouvrière en ce qui le concerne). Le microcosme familial est comme un miroir, un reflet des tensions et contradictions de la société dans son ensemble. Il s'agit, dès lors, de systématiser l'observation directe des situations sociales singulières pour remonter ensuite à la société toute entière. Son emploi systématique des analyses monographiques descriptives et singulières comme unités de base ${ }^{10}$ va toutefois nourrir les reproches notamment des statisticiens Alfred de Foville et Maurice Block qui jugent la méthode trop incertaine et aléatoire. Des socio-

I0 Sa démarche scientifique consiste à construire des indicateurs sociaux basés sur l'économie domestique des familles ouvrières. 
logues, Durkheim en premier mais aussi René Worms ${ }^{11}$ ne la condamnent pas mais mettent en doute son intérêt. En circonscrivant l'analyse aux seules études de cas, ils contestent une posture qui empêche la montée en généralité. Ils lui reprochent de décrire sans expliquer. En somme, de ne pas faire vraiment de la science ${ }^{12}$. Face au positivisme sociologique prônant l'objectivation des connaissances dans l'étude des faits sociaux, Le Play préconise à l'opposé une analyse qualitative et sensible de cas typiques représentatifs de la classe ouvrière naissante. Certes, le recours aux enquêtes monographiques n'est pas entièrement nouveau ${ }^{13}$, mais Le Play est le premier qui «systématise sa démarche d'investigation» (Savoye, 1994, p. 54). Son œuvre sera poursuivie par des sociologues réunis au sein de la Société internationale des études pratiques d'économie sociale fondée en i856. Henri de Tourville (I883) notamment effectue, sur la base d'une sociologie monographique de grande ampleur, une nomenclature des faits sociaux. Émile Cheysson (I896) mobilise les cadres monographiques pour l'étude de la commune et de l'atelier ${ }^{14}$. La sociologie leplaysienne est interventionniste car elle étudie les faits sociaux dans le contexte de l'ici et maintenant (Hess, 198ı, p. 94). En voulant faire de l'ingénieur un acteur du social, Le Play revendique l'opérationnalité de

II Worms écrit à ce propos : «Ce qu'on doit seulement se demander, c'est si les résultats ainsi obtenus sont suffisamment généraux pour les besoins de la science. Celle-ci vise à formuler des vues et des lois d'ensemble, et on sera porté à douter que des données relatives à telle famille, à tel atelier, voire même à telle commune isolément considérée, puissent être étendues à toute une société, servir à constituer la science sociale proprement dite» (1918 [1904], p. 124).

12 Pour Savoye et Audren, les raisons de ces critiques sont très contextuelles. La période se prête à une survalorisation de la «théorisation abstraite - caractéristique de la démarche philosophique traditionnelle - au détriment de la réflexion fondée sur une connaissance directe, sensible, de faits concrets observés spécialement à des fins d'étude» (2008, p. 19). Mais une autre critique, plus politique et probablement plus radicale (Kalaora et Savoye parlent de «conspiration du silence») explique aussi le faible écho que les travaux de Le Play rencontreront par la suite. En plein élan républicain, ses idées mâtinées de catholicisme conservateur et son appel au retour de la famille traditionnelle sont mal perçus (Le Play se réclame notamment du «Décalogue» explicitant les dix commandements moraux et religieux et appelle de ses vœux le retour de la «famille souche»).

13 Voir Alexandre Parent-Duchatelet sur la prostitution en 1836, Tristan Flora sur le prolétariat anglais en 1840, Adophe Focillon sur les savonniers en 1859.

14 Pour remédier aux critiques formulées contre la méthode monographique, Cheysson suggèrera notamment d'associer la statistique à la monographie. 
la sociologie. Il réalise une sociologie sensible dans la mesure où il se place au plus proche des acteurs supposés représentatifs de la société en mutation. Sa sociologie se veut résolument micrologique en s'appuyant sur l'étude approfondie de faits concrets observés depuis l'intérieur. Comme le rappelle Françoise Arnault (1984), cette posture inscrit une véritable rupture avec la sociologie dominante qui s'impose dans les milieux universitaires français.

\section{I.2. L'EMPIRISME AMÉRICAIN}

\section{ET L'AMBIVALENCE DE LA SOCIOLOGIE DE TERRAIN}

La genèse de la sociologie d'intervention nous conduit certes jusquaux conceptions le playsiennes, mais celles-ci ne sont, pour autant, que «les maillons d'une chaîne » (Savoye, 1979, p. 205) qui commence avec les premières études du monde ouvrier. Pour suivre l'évolution de la sociologie d'intervention, souligne Herreros (2008, p. 84), il convient de se tourner vers la tradition anglosaxonne et notamment américaine. Le prolongement se situe pour l'essentiel dans les formes d'enquêtes qualitatives qui seront utilisées par les chercheurs pour aborder et interpréter le monde social. Dans la ligne de Le Play va s'illustrer notamment le courant empiriste des sociologues américains de l'école de Chicago. Dès son origine, celle-ci cultive une posture pragmatique et un intérêt aigu pour les problèmes concrets de la société. Les sociologues américains sont, dès le début, happés par les relations interindividuelles. Si la philosophie sociale, inspirée des pensées de Montesquieu, Montaigne et plus globalement de l'esprit des Lumières, s'implante sur le continent américain, elle se tourne rapidement vers une pensée plus concrète et moins spéculative. Albion Small insiste tout particulièrement auprès de ses étudiants pour qu'ils multiplient les recherches actives. L'ouvrage qu'il publie en I894 avec George Vincent - une «introduction to the study of society » - comprend notamment deux chapitres consacrés spécifiquement à la conduite empirique de la sociologie. Charles Horton Cooley est convaincu que la société ne peut se comprendre sans une prise en compte du sujet pensant interprétant le monde. Ce regard conduit cet auteur à se démarquer d'une lecture scientifique qui ne soit pas aussi psychologique ${ }^{15}$. 
Ces différents auteurs ${ }^{16}$ vont s'appliquer à développer une microsociologie en concentrant leurs recherches sur des groupes restreints (quartiers, petites villes, communautés) connaissant de profondes difficultés d'intégration liées à l'immigration, l'alcoolisme, la drogue, la pauvreté, etc. Pour eux, l'étude des hommes doit partir de l'observation et de la description behavioriste pour rechercher «la signification que les acteurs sociaux donnent de leurs comportements et de la situation dans laquelle ils se trouvent» (Simon, 199I, p. 435). En positionnant le chercheur au plus près des acteurs, tous ces travaux ont un même fondement: ils attribuent à l'expérience individuelle une place active dans la compréhension sociologique. Ils marquent ainsi le point de départ d'une tradition empiriste caractéristique des origines de la sociologie américaine ${ }^{17}$. Plus tard, William Isaac Thomas s'illustre par ses premières études de masse contenant d’abondantes histoires de vie et des biographies extrêmement précises. En I92 I, Robert Park publie avec Ernest Burgess une Introduction to the science of sociology qui sera la référence des générations d'étudiants soucieux de réaliser ce que Park appelle des «vraies recherches de terrain $»^{18}$. Mais contrairement à une posture interventionniste plus aboutie, l'observation directe préconisée par les chercheurs de l'école de Chicago n'implique pas une immersion participante du sociologue, bien au contraire. Rares en réalité sont les sociologues qui vont réellement participer à la vie des groupes qu'ils étudient ${ }^{19}$. En fait, l'observa-

16 Ajoutons William Edward Burghardt Du Bois, premier sociologue noir américain, qui publiera en 1899 «The Philadelphia Negro».

17 L'aura de la sociologie américaine n'est pas, toutefois, le seul fait de ces premiers empiristes. D'autres auteurs issus de courants intellectuels et philosophiques divers vont apporter aussi leurs pierres à l'édifice. Pour le pragmatiste John Dewey, la réalité est à rechercher dans ses conséquences réelles et mesurables dans le monde social. Pour George H. Mead, les relations se construisent par le jeu des interprétations et des symboles qui rapprochent et éloignent les individus. Pour comprendre ce sens, les interactionnistes considèrent que le chercheur doit devenir lui-même un acteur social en participant activement à la vie des groupes qu'il étudie.

18 Ces travaux vont inspirer une série de monographies réalisées par ses élèves, Everett Stonequist sur le «marginal man», en 1937, Nels Anderson sur les «hobos», en 1923, Louis Wirth sur les juifs, en 1925, Harvey Zorbaugh sur les résidents des quartiers chics, en 1929.

19 C'est toutefois le cas de Frederic Thrasher sur les gangs en 1927, de Paul G. Cressey étudiant les Taxi-dance Halls en 1932 ou encore de William Foote Whyte s'immergeant dans le quartier italien en 1943. 
tion pratiquée est plus active que participante (Chapoulie [2000] la qualifie de «diffuse »). À une époque où la sociologie doit encore affirmer sa scientificité, on craint qu’un interventionnisme trop poussé nuise à la légitimité universitaire de la sociologie américaine. Aussi, l’objectivité du sociologue est-elle revendiquée pour le prémunir d’une posture qui serait jugée trop journalistique et militante. Ce n'est quaprès les années 1940 que le recours à l'observation «analytique» (Chapoulie, ibid.) devient plus systématique. Pour mener leurs études respectives sur les fumeurs de Marijuana, les déficients mentaux ou le milieu médical conduites dans les années 1950-1960, Howard Becker, Erwing Goffman et Anselm Strauss vont s'immerger en situation concrète durant de longues périodes. Notons que cette technique d'immersion sur le long terme se prolongera jusqu'à aujourd'hui et se retrouve dans des travaux plus récents chez Stéphane Beaud et Michel Pialoux (1999), Loïc Wacquant (200I), Florence Weber (200I), Michel Pinçon et Monique Pinçon-Charlot (2007) ou encore Dominique Schnapper (2010). Cette dernière utilise l'expression de «participation observante» (2OII) pour caractériser son positionnement de «sociologue indigène» au Conseil constitutionnel ${ }^{20}$. Progressivement, l'empirisme américain s'institutionnalise. Pour autant, la sociologie américaine n’est pas dénuée d’ambivalences et il convient aussi de modérer la portée réelle de ses résultats dont la tendance à un certain conformisme de la pensée a été critiquée dès les années 1950 par de nombreux sociologues français. Dans son article consacré au Human Engineering, Michel Crozier dénonce ainsi une sorte de «caricature de science» (195 I, p. 58) qui, à force de répéter que la solution est dans la participation, les relations humaines et l'écoute du chef envers ses salariés, finit par instituer comme une évidence que toute forme de révolte et d'opposition est contre nature, et que l'homme n'aspire qu'à une chose : entretenir de bonnes relations avec ses congénères. Ce ne sont pas les méthodes du Human Engineering qu'il convient de reconsidérer, précise Crozier, mais davantage l'esprit qui préside à leur emploi ; un esprit qui «consiste à traiter l'opposition comme une névrose ou une maladie» (1951,p. 65). D’autres réserves sont émises par des

20 Elle se qualifie elle-même d'indigène dans la mesure où elle a siégé neuf années au sein du Conseil constitutionnel qu'elle a en même temps analysé de l'intérieur. 
sociologues tels Alain Touraine et Raymond Aron regrettant «la faible prise en compte de la dimension historique des faits sociaux» (Chapoulie, I99I, p. 344). Le contexte social extérieur à l'atelier et au groupe étudié leur paraît insuffisamment pris en compte conduisant la sociologie américaine à produire «une masse d'enquêtes à caractère descriptif qui semblent dépourvues de cadres conceptuels explicites » (ibid.). En outre, l'important soutien financier dont bénéficient les sociologues outre-Atlantique confirme un peu plus encore le sentiment que leurs études sont commanditées à des fins de manipulation par le «big business» (Crozier, I95 I). On le voit, si les enquêtes de terrain témoignent de la capacité de la sociologie américaine à mieux comprendre des individus en situation, elles ne parviennent pas, pour autant, à annuler ses propres contradictions internes que les sociologues français notamment souligneront à maintes reprises. Ce qui pose problème, dès lors que le sociologue revendique son attachement à l'empirisme, c'est bien son aptitude à préserver son autonomie face aux pressions qui s'exercent sur lui, c'est aussi sa capacité à dépasser le stade de l'analyse d'une situation singulière pour adopter une vision plus globale. Si ces limites sont bien présentes, ne faut-il pas toutefois les considérer comme un mal nécessaire pour réellement parvenir à comprendre et expliquer les situations sociales et permettre aux acteurs d’agir en conséquence?

\section{CARACTÉRISTIQUES DE LA SOCIOLOGIE D'INTERVENTION CONTEMPORAINE}

Cette manière de percevoir le métier de sociologue va connaître en France un développement certes plus modeste ${ }^{21}$ mais bien réel. L'objectif de cet article n'étant pas de présenter les principales caractéristiques des théories françaises d'intervention - caractéristiques abondamment explicitées et discutées par ailleurs - notre intention est bien davantage de souligner les points de convergences théoriques et méthodologiques susceptibles de donner à la sociologie d'intervention un cadre commun et unifié.

21 Chapoulie souligne «une direction à peine explorée» des enquêtes de terrain dans la sociologie française (2000, p. 22). 


\section{I. MAÏEUTIQUE ET PRINCIPE COLLECTIF D'AUTOANALYSE}

L’analyse institutionnelle (AI), portée par le groupe vincennois constitué de René Loureau, George Lapassade et Rémi Hess, propose une lecture politique de la société. En quarante années d’activités, elle va élaborer sa propre méthode d'intervention: la socioanalyse. Définie par Lapassade comme la «méthode par laquelle un groupe d'analystes, à la demande d'une organisation sociale, institue dans cette organisation un processus collectif d’autoanalyse », (Hess et Savoye, 1993, p. 97), elle cherche à mettre à jour les processus institutionnels cachés «d'occultation, de scotomisation et de refoulement» (Hess et Authier, 1994, p. 78)22. Affirmant l'incontournable subjectivité du chercheur (ce qu'on a pu appeler «l'effet Heisenberg » décrivant les mécanismes de transfert et de contre-transfert), la socioanalyse vise à susciter l'autogestion de l'intervention par le groupe. Ce dernier, passant d'objet à sujet, met en place son programme d’analyse, ses propres dates, horaires et fréquences de travail. Cet effort de clarification s'accompagne d'une prise de conscience par les auteurs eux-mêmes du véritable ordre institué. Mais c'est aussi cet excès de vulgarisation et le manque de plus en plus flagrant de réflexion épistémologique qui perdront l’analyse institutionnelle. En revendiquant une sorte «d'ailleurs sociologique» s'inscrivant résolument hors du système académique, l'approche socioanalytique de l'aveu de Lapassade (1996) finira par se dissoudre dans une multitude de microsociologies incapables de lui donner consistance. Ce qui est révélation de l’ordre institué dans l'AI est révélation des mouvements sociaux dans l'analyse tourainienne. Pour le fondateur de l'intervention sociologique, le rôle du sociologue est de révéler les enjeux des conflits qui attestent de l'existence des mouvements sociaux, lesquels constituent le «sens le plus élevé» des conduites collectives (Touraine, 1978, p. 30I). Pour ce faire, «l'intervention doit être aussi proche que possible de l'action» (ibid., p. 205). Cela conduit le chercheur à dépasser le discours idéologique pour saisir les acteurs dans la profondeur de leur action militante. Pour l'intervention sociologique, le chercheur agit activement et

22 Selon le principe de la règle du «tout dire», il s'agit de révéler au grand jour la vraie vie de l'organisation avec ses propres codes et ses propres règles de fonctionnement (ce que les institutionnalistes appellent l'«agorisme» en opposition au «cryptisme» ou l'art du secret). 
personnellement pour amener le groupe d'analyse - les acteurs qui constituent ce groupe ne sont pas en général les dirigeants mais bien plutôt les acteurs de la base entrant en lutte contre un système qu'ils contestent - à prendre conscience de la nature de ses rapports sociaux ${ }^{23}$. Cela passe notamment par des confrontations de points de vue avec des interlocuteurs extérieurs habilement choisis par les acteurs eux-mêmes et par les chercheurs dont «le premier but est de développer cette conscience de l'acteur» (Touraine, 1984, p. 206). Le principe maïeutique sous-tendu vise donc à pousser chaque individu à accoucher de sa conscience (Cousin et Rui, 2010, p. 9). Comme le clame Touraine, cela n'est possible que si les individus participent pleinement à l'interaction avec les chercheurs. Contrairement à d'autres formes d'intervention, l'intervention tourainienne ne vise pas nécessairement une meilleure adaptation ou la conduite du changement; elle se veut essentiellement au service de la connaissance (Touraine, 1978, p. 262 et p. 278). Reste que cette connaissance peut se révéler utile aux acteurs du terrain. Comme les théories d’intervention précédemment exposées, la méthode développée par l’actionnalisme tourainien veut susciter la capacité autoréflexive des individus : «Par le biais de la méthode, les acteurs sont invités à entreprendre un travail de réflexion, voire d’introspection, qui place en son centre l'analyse de la manière dont ils lisent et interprètent le monde social et qui interroge leur capacité à agir et intervenir sur ce monde » (Cousin et Rui, 20II, p. 514). En recherchant l'autoanalyse du groupe par une implication personnelle du chercheur, l'intervention sociologique affiche ses similitudes avec l'ensemble des théories sociologiques d'intervention. Pour les tenants de l'analyse stratégique, la posture n'est pas radicalement différente. Il s’agit de rendre compte empiriquement de la nature et des règles du jeu qui structurent les relations entre les acteurs sociaux. Scientifique par ses procédures ${ }^{24}$, l’analyse stra-

23 La constitution quelque peu artificielle du groupe d'analyse sera l'objet de nombreuses critiques. Comment créer un dispositif le plus proche possible de l'action à partir d'un regroupement d'individus n'ayant pas à l'origine de rapports réels entre eux? Cette décontextualisation, soulignent Olivier Cousin et Sandrine Riu, tend à «substituer à l'action effective une situation d'action fictive» (2010, p.96). Dès lors, note Danilo Martucelli, «l'intervention risque de n'être rien d'autre que le résultat du travail d'un groupe, un résultat dont on ne peut connaître la portée générale» (199|, p.3).

24 L'analyse stratégique se qualifie de «science procédurale» (Friedberg, 1997 [1993], p.32I) dans la 
tégique s’affirme aussi interventionniste. La méthode développée est «clinique » car, au chevet de l'organisation, elle vise à diagnostiquer son état de santé et à en rendre compte aux acteurs concernés. Dans cette prise de conscience de phénomènes dont les acteurs sont aussi les auteurs, l’analyse stratégique «suggère des "boucles autoentretenues" où chacun participe de la production de ce dont il se plaint» (Herreros, 2009, p. 65). Il en découle, précise Erhard Friedberg, un rapport à la théorie très différent de la norme académique dans la mesure où la théorie tire sa pertinence essentiellement de sa capacité à nourrir une confrontation constante avec le terrain : «la théorie elle-même ne devient concrète que rapportée à une situation empirique » (1997 [1993], p. 300). C'est essentiellement dans l'annexe de l'ouvrage fondateur de 1977 et dans deux ouvrages écrits par Friedberg en 1988 (1972) et en 1993 (réédité en 1997) qu’on trouve quelques éléments méthodologiques. Face à des microsituations complexes, les auteurs identifient ainsi trois temps dans l'analyse. Dans un premier temps, le sociologue doit pouvoir «se ménager une position de recul et de distance critique lui permettant de rompre avec la réalité sensible, avec les catégories de sens commun» (1977, p. 455). Mais cette posture, reconnaissent les auteurs, est en réalité bien difficile à tenir. Aussi (c'est le second temps), le sociologue doit savoir rapidement «quitter sa position d'observateur détaché et extérieur au champ pour opérer un détour par l'intériorité des acteurs» (ibid., p. 456). Le sens profond des relations interindividuelles ne se laissant pas facilement dévoilé, il s'agit d'entrer de «plain-pied» dans la situation et se mettre à la place des acteurs pour comprendre leurs logiques internes. Ce retour vers l'intérieur, précise Friedberg, révèle une posture éminemment clinique, nécessairement contingente et abolissant temporairement toute distance entre le sociologue et les acteurs en situation (1997 [1993], p. 303)25. Ce n'est que plus tard (troisième temps) que le sociologue

mesure où sa scientificité ne se situe pas dans sa logique interne mais réside dans les procédures méthodologiques qu'elle mobilise. Pour ses auteurs, le caractère scientifique des analyses est en réalité «fonction du respect d'une procédure d'enquête qui doit garantir un recueil et un traitement des données aussi ouverts, systématiques et honnêtes que possible» (ibid.). Le respect de cette procédure doit prémunir le chercheur de l'exercice débridé et hors de contrôle de sa subjectivité.

25 Ce retour vers l'intérieur constitue une «abolition de la coupure épistémologique»entre la théorie et la pratique (ibid., p. 306) car il cherche à empêcher le cloisonnement du chercheur dans sa discipline et dans une supposée posture d'objectivation des connaissances. 
doit de nouveau «reconquérir son extériorité en confrontant et comparant les unes aux autres les multiples rationalités ou stratégies contingentes qu'il aura observées » $\left(1977\right.$, p. 457) ${ }^{26}$. À ce stade de ses recherches, il cherche à mesurer les écarts entre ce qui devrait se passer «normalement» et la réalité - les auteurs parlent de «raisonnement sur les écarts» (ibid.). Quand l'opportunité le lui permet, le chercheur peut tenter une progressive montée en généralité qui se fait notamment en comparant les résultats des différentes études ayant porté sur des champs proches. Toutefois, cette montée en généralité ne constitue pas à proprement parler la finalité de l’analyse stratégique. Comme le rappelle Friedberg, ce n'est pas «parce qu'on ne peut pas extrapoler facilement les résultats d'une étude particulière que son intérêt est nécessairement limité » (1997 [1993], p. 325). Pour l’analyse stratégique, ce n’est pas là l'essentiel.

Dans sa thèse de 1979 qu'il consacre à la genèse de la sociologie d'intervention, Savoye en définit utilement quelques contours. La posture d'intervention marque sa singularité par la proximité qu'elle entend nouer avec la population étudiée, cette proximité ayant pour fonction de déclencher un «effet analyseur» (Savoye, 1979, p. 2 et p. I3). Dans cette sociologie de terrain, ce qui compte, «c'est le rapport direct institué entre le sociologue et la population qu'il étudie» (ibid., p. 3). Faisant du sociologue «une partie prenante du processus de changement visé» (ibid., p. 7), l’auteur définit l'intervention comme l'action de «venir entre», de réaliser une sorte «d'incursion» dans les rapports sociaux (ibid.). Le sociologue est celui qui «sur-vient par rapport à un état pré-existant» (Dubost, 1987, p. 152), en suscitant chez les acteurs l'autoanalyse et leur pleine implication dans la recherche des moyens d'action. Dans la partie suivante, nous allons tenter de préciser ce qui peut contribuer à mieux singulariser la sociologie d'intervention contemporaine.

26 Parlant d'agnosticisme, Friedberg explique que le sociologue, autant que possible, doit éviter de prendre parti et veiller à garder une certaine équidistance dans l'interprétation des faits (1997 [1993], p. 307). Mais peut-on vraiment croire que le sociologue parvienne si facilement à recouvrer son extériorité et adopter de nouveau une totale objectivité? 


\subsection{LE SOCIOLOGUE DANS L'ARÈNE}

L'étude des phénomènes sociaux dans le vif de leur actualité

Qu'est-ce que la sociologie d'intervention aujourd'hui? Comment peuton la caractériser? La sociologie d'intervention, explique Guy Minguet, est un exercice professionnel, épistémologique, méthodologique, postural qui conduit le sociologue praticien à se positionner, sur sollicitation d'un demandeur, au cœur d’une situation afin de l'améliorer (200I, p. 2I). Le sociologue doit s'immerger dans la situation qu'il analyse. Par le mot «intervention», il s'agit de penser le sociologue comme un scientifique capable de faire évoluer avec les acteurs sujets de l'étude, les situations dans lesquelles ils interagissent pour atténuer collectivement les difficultés qu'ils rencontrent. En fait, on peut affirmer que toute sociologie est une intervention dès lors qu'il s'agit (et cela arrive à tout sociologue) de confronter les analyses d'une enquête aux points de vue des premiers concernés. Pour autant, cette confrontation peut être de nature différente: directe, sous-jacente ou masquée. Comme le souligne François Dubet, dans la nature des relations entre le chercheur et les acteurs, «tout est affaire de degré» (200I, p. 90). Pour le sociologue d'intervention, la confrontation des idées avec les acteurs est directe et permanente. Par sociologie d'intervention, il faut donc entendre «une pratique spécifique de la discipline conduisant le sociologue à s'immerger, sur (auto)sollicitation, dans une microsituation, afin d'en proposer une lecture dont les effets (de contenu ou de processus) pourraient participer d'une transformation de ladite situation » (Herreros, 200I, p. 273). La posture du sociologue consiste donc à se placer parmi les acteurs, dans l'intention de leur apporter «une plus-value cognitive mobilisable pour l'action» (ibid.). Le but escompté est de découvrir des univers sociaux, les dimensions cachées des phénomènes sociaux que la perspective quantitativiste, statistique voire qualitative (le recours aux entretiens par exemple) ne permet pas toujours de restituer dans son intégralité. Dans cet article, nous ne prétendons nullement proposer une définition exhaustive de la sociologie d'intervention. Bien même que nous le souhaiterions, la polysémie des termes et la diversité des sensibilités sous-jacentes auraient tôt fait de nous en dissuader. Cette tentative de délimiter et de mieux caractériser cette posture 
sociologique répond avant tout à une volonté de contribuer à sa meilleure reconnaissance dans le champ académique et scientifique.

Afficher sa préférence pour une sociologie de terrain n'est pas, comme l'exemple de la socioanalyse le montre, dénué de risques. L'écueil d’une dilution de ses fondements dans une microsociologie trop descriptive n'est jamais très loin. Aussi le sociologue doit-il s'astreindre à un travail permanent de clarification et d'autoréflexivité. De la même manière que la servitude suppose une volonté minimale d'obéir, l'intervention trouve sa raison d'être dans l'existence chez les individus d'une volonté minimale de coopérer. Pour pouvoir mettre en travail une situation, le sociologue doit être sûr que les acteurs en présence, ou tout au moins un nombre suffisant d'entre eux, expriment une réelle demande sociale. Celle-ci, entendue comme «le système d’attentes de la société à l'égard des problèmes quotidiens qui la sollicitent aujourd'hui» (Castel,2004, p. 70) peut émaner des dirigeants - les commanditaires - mais aussi des salariés confrontés à des situations de malaises, de tensions voire de conflits. Comme le soulignent Monique Legrand et Didier Vrancken (2004, p. I55), un travail d'explicitation de cette demande est d'ailleurs bien souvent nécessaire en amont et durant l'intervention. Il n'est pas rare en effet que le sociologue soit sujet, en certaines circonstances, à des formes d'instrumentalisation qui peuvent être le fait des commanditaires ou des acteurs du terrain. Cette instrumentalisation peut prendre la forme d'une caution que constituerait le sociologue pour légitimer, à son insu, les prises de décisions de certains acteurs, voire pour disqualifier les parties adverses ${ }^{27}$. La sociologie d'intervention vient également questionner le sens commun. S’il est d'usage, pour les auteurs de l'ouvrage Le Métier de sociologue (Bourdieu, Chamboredon, Passeron, 1983) d’instituer une véritable rupture avec le sens commun ${ }^{28}$, d’autres auteurs comme François Chazel (20I I) sont plus nuancés et prônent davantage sa progressive mise à distance. C’est aussi une préconisation de Durkheim invitant la sociologie à partir des

27 Dans le premier cas, François Pichault parle de stratégies de légitimation, dans le second cas, de stratégies de disqualification (2006, p. 78).

28 Pour les auteurs, le sens commun relève d'une «sociologie spontanée» (1983, p. 27) dont la vertu explicative n'est que «vœu naïf» et «illusion» (ibid., p. 32-33). 
«notions vulgaires» (1999 [1895], p. 34) pour opérer progressivement leur mise à distance par le raisonnement scientifique - «Le concept vulgaire n'est pas inutile ou savant, en réalité, il sert d'indicateur», écrit-il (ibid.,p. 37). Pour la sociologie d'intervention, le sens commun est considéré comme essentiel car producteur de connaissances permettant une amélioration à terme de la situation. Ce qui, chez Bourdieu, est appelé sens commun ou doxa, est considéré comme un savoir chez Alfred Schütz, une compétence chez Luc Boltanski ou encore une aptitude à la réflexivité chez Anthony Giddens. Pour ce dernier, la relation entre la sociologie et les individus relève d'une «double herméneutique» (1994, p. 24). D’une part, la science fournit des connaissances et des informations sur la vie sociale et remet en cause les «concepts des agents profanes» (ibid.). D’autre part, ces connaissances forgées dans le métalangage des sciences sociales sont réinjectées dans la société et réappropriées par les acteurs. Il s'ensuit «un va-et-vient entre l'univers de la vie sociale et le savoir sociologique et, dans ce processus, le savoir sociologique se modèle et remodèle l'univers social» (ibid.). Sur le terrain, le chercheur se réfère à la maïeutique considérant que «personne n'est jamais qu'ignorance. Le savoir que chacun possède, qu'il affleure ou soit enfoui, ne demande qu’à être découvert » (Herreros, 2009, p. I45). En ce sens et contrairement à la conception bourdieusienne - tout au moins avant La Misère du monde (1993) - l'approche maïeutique refuse toute «asymétrie» instaurant une supériorité des savoirs scientifiques sur les savoirs communs (Boltanski et Thévenot, 1991, p. 63). L'essence même de la sociologie d'intervention est dans la combinaison des champs scientifique et «profane», écrit Yves Gilbert (2009, p. 255-256), considérant que l'individu est réflexif - cette réflexivité s'inscrit dans la «conscience pratique»si on suit Giddens (1987 [1984]) et, à un niveau plus conceptuel, dans la «conscience discursive » - et détient, à des degrés divers, suffisamment d'intelligence pour identifier la solution qui est pour lui la moins mauvaise ou la plus acceptable (Dupuy, 2004, p. I22). Parfois perçu comme un expert, le sociologue entend de ce fait marquer sa différence avec la posture construite et très normative de l'expertise (Lenoir et al., 1989). Considérant que l'expert analyse une situation à travers son propre prisme (Enriquez, 2002) - jugé trop spécialisé, il ne peut 
restituer toute la réalité d'une situation - le sociologue construit sa connaissance avec les individus «profanes». Le capital scientifique du chercheur et le capital spécifique de l'acteur de terrain s'enrichissent mutuellement : comme l'écrit Rémi Hess «le savoir social se produit collectivement par les acteurs sociaux» (I98I, p. I2-I3). S’il convient de considérer avec Schnapper que «les méthodes ne valent que pour les résultats qu'elles permettent d'obtenir» (20II, p. I), cela signifie que l'approche sociologique tend aussi à privilégier une forme de métissage méthodologique ${ }^{29}$ amenant le praticien sociologue à explorer toutes les branches de la sociologie depuis les approches les plus classiques jusqu'à celles empruntant les voies de la psychologie et de la clinique. Jean-Michel Berthelot le souligne à juste titre: «Tout découpage est partiellement arbitraire», ajoutant que «les frontières entre disciplines sont, au regard de leur histoire, si poreuses et fluctuantes que l'on passe de l'une à l'autre sans véritable seuil» (200I, p. 203). De ce fait, «aller au plus près du vécu des acteurs conduit à remettre en question les frontières entre psychologie et sociologie, extériorité et intériorité, objectivité et subjectivité, réalité et représentation»(De Gaulejac, Hanique et Roche, 20I2, p. 66) ${ }^{30}$. L'intérêt d'une approche pluridisciplinaire est de permettre d'ouvrir plusieurs tiroirs situés à des niveaux différents. Avec la sociologie clinique en particulier, le but, in fine, n'est pas de refaire la sociologie mais bien de la penser différemment ${ }^{31}$. C'est ce que propose Bertrand Ravon en préconisant une lecture clinique du social pour comprendre la société et ses malaises sociaux (2009, p. I54). Quand l'analyse est bien avancée voire achevée, la restitution par le sociologue de sa «représentation du réel» (Uhalde, 200 I p.400) l'expose à la contestation des acteurs - Vrancken (1995) parle de leur «indocilité».

29 Dans cette pensée plastique, «ce qui est métis n'est pas perdu ou dilué, mais redécliné» (Herreros, 2008, p. 269).

30 Cette dimension psychologique dans la sociologie classique est du reste présente dès les origines des sciences sociales. Mauss n'appelle-t-il pas lui-aussi de ses vœux une «sociologie psychologique» (1968) qui analyse le rapport des faits psychiques et des faits matériels dans la société, ce rapport constituant, à ses yeux, une partie essentielle de la sociologie?

31 C'est d'ailleurs par ce recours à l'étude clinique comme méthode qualitative que Friedberg (1993, p. 312) entend se détacher de la standardisation des méthodes d'enquêtes quantitatives. 
Dans ce débat indispensable, le chercheur comme l'acteur ont leur mot à dire, le premier ayant en plus du second cette capacité éprouvée de faire parler le terrain. Seulement, sa position n'est pas surplombante ni prééminente. C'est avec l'ensemble des acteurs concernés et grâce à eux qu'il parvient à tirer du mieux et du tangible de la situation étudiée.

Une telle représentation du métier de sociologue conduit à questionner un point fondamental : l'objectivité du sociologue immergé dans la situation qu'il analyse et sur laquelle il agit par sa seule présence (c'est le classique «paradoxe de l'observateur » décrit par William Labov) ${ }^{32}$. Giddens l'affirme dans sa théorie de la structuration: le sociologue fabrique du social en contribuant à modifier l'objet qu'il étudie. Tandis qu'une partie de la sociologie entretient avec les phénomènes sociaux un rapport distancié et neutre, la sociologie d'intervention assume un rapport spontané et moins intemporel avec les situations concrètes, les phénomènes sociaux étant appréhendés dans le vif de leur actualité et de leurs particularismes ${ }^{33}$. D’un côté, le sociologue recherche, par l'observation, la mise à l'épreuve d'hypothèses préalablement posées en mobilisant l'arsenal méthodologique. De l'autre côté, le sociologue s'attache à mettre en lumière les dimensions cachées des phénomènes sociaux en s'émancipant des catégories sociales préconstruites ${ }^{34}$. Ici, «les modèles théoriques se trouvent sans cesse enrichis par le contact avec les faits concrets » (Kaloara et Savoye, 1989, p. 267). À plus forte raison parce que l'intervention met le sociologue dans «la fosse de Daniel », elle rend difficilement tenable une position de réelle objectivité et de mise à distance à l'objet. Comme le disent Yves Gilbert et Brigitte Baldelli, «il faut définitivement tordre le cou au mythe de l'objecti-

32 La question de l'objectivité du chercheur immergé a été maintes fois soulevée par ceux notamment qui ont le plus critiqué la sociologie empirique américaine, considérant que la proximité du sociologue avec ses interlocuteurs l'influençait nécessairement au point de remettre en cause la scientificité de ses analyses.

33 Elle est davantage ce qu'Edgar Morin appelle dans La Rumeur d'Orléans (1970) une «sociologie du présent».

34 Le sociologue de terrain, écrit Jean-Michel Chapoulie, est «maître du codage de ce qu'il observe» (2000, p. 20). 
vité du chercheur en sciences sociales » ${ }^{35}$ (20I I, p. I4). Le sociologue projette presque inévitablement, rappelle Serge Paugam, une partie de lui-même dans ses recherches (2008, p. 19). Dans cette relation de sujet à sujet, cela conduit à dire que le sociologue n'est jamais dans une complète distanciation avec son objet. Il «épouse toujours une cause » (Uhalde, 200I, p. 400) ${ }^{36}$. Reste que cette cause ne doit pas le faire basculer dans un subjectivisme aveugle. Pour éviter cet écueil, l'éthique du sociologue constitue probablement son principal repère, son «butoir essentiel» (Herreros, 2009, p. 237). Pour reprendre les propos de Richard Rorty (1994), l'éthique peut consister à minimiser les petites choses particulières qui séparent les individus dans le but de tendre vers le compromis et l'entente collective ${ }^{37}$. C'est là une utilité sociale forte du sociologue qui se mesure aux effets positifs que ses actions sont susceptibles de produire auprès des acteurs en situation. Mais on ne peut nier, il est vrai, que ces «effets positifs » revêtent, dans l'appréciation du sociologue, un caractère très subjectif, voire normatif, qui nous renvoie aux critiques formulées par Crozier sur le Human Engineering. On le voit, la sociologie d'intervention, si elle veut vivre et exister, ne doit jamais faire l'économie d’une réflexion critique sur ses propres fondements épistémologiques.

35 Pour neutraliser le subjectivisme de l'enquêteur, Bourdieu a pu préconiser, à une époque, de recourir au système de la proximité (ou géométrie) sociale (1993, p. 1395) entre l'enquêteur et l'enquété, mais au risque, note Jacques Hamel (2007, p.52), de créer entre eux une trop forte familiarité nuisible à la production de connaissances.

36 Rejetant toute pensée objective qu'ils jugent impossible, certains auteurs adoptent même une posture très radicale. C'est le cas de Richard Rorty qui revendique un ethnocentrisme assumé, privilégiant son «propre» groupe, sa communauté, plutôt que de prétendre s'en détacher. C'est même cette appartenance qui lui permettrait de pouvoir réellement analyser sa communauté.

37 Ce que Rorty appelle la «commune humanité» (ibid., p. 126). 


\section{CONCLUSION}

\section{Utilité locale et montée en généralité}

Comme nous avons essayé de le démontrer tout au long de cet article, les théories sociologiques d'intervention, bien que de sensibilités parfois très différentes, reposent néanmoins sur un socle conceptuel et méthodologique commun. Certes, il y a des différences d’appréciation sur la demande sociale, la posture du sociologue ou encore les finalités. Certains auteurs entendent marquer leur singularité. C’est le cas de Touraine pour qui la demande n'est nullement un préalable à l'intervention sociologique, seul le mouvement social importe. D’autres tentent de minimiser l'implication du sociologue qui doit, selon Friedberg, faire œuvre, autant que possible, d’agnosticisme. Certes, la transdisciplinarité n’a pas la préférence de Touraine plutôt attaché au monisme disciplinaire, ni de Friedberg. Quant à la finalité de la sociologie, elle diffère quelque peu d'un auteur à l'autre. Elle consiste à mesurer le poids de l'histoire dans l'esprit tourainien, à mieux réguler les rapports de pouvoirs pour l'analyse stratégique, ou à libérer l'acteur d'un système aliénant pour l'analyse institutionnelle. Elle réside encore dans la recherche d'une situation meilleure au moyen d'une «anthropologie d'intervention» (Herreros, 2009). Cela étant, au-delà de ces clivages, toutes ces sociologies exposées précédemment partagent néanmoins une même manière d’aborder la «réalité chaude» (Hess, 198I) du monde social où «le chercheur et son objet se trouvent dans une relation de confrontation productive» (ibid., p. 200). En se réclamant des mêmes principes posturaux, en appelant au renoncement de toute rupture épistémologique entre les savoirs, de toute forme de cloisonnement entre les niveaux de réflexivité, elles dessinent pour le sociologue du vécu des contours et une identité qui lui donnent sa consistance. Certes, la sociologie d'intervention est un paysage à relief multiple (Vrancken, 200I, p. 312) mais la conception du rôle que doit jouer le sociologue s'inscrit résolument dans l'action locale en cherchant à offrir aux acteurs du terrain une plus grande intelligibilité de leurs comportements sociaux. Dans cette mise au travail de la situation, le sociologue réduit les distances avec les acteurs pour susciter leur autoréflexivité, par le questionnement, le dérangement voire l'agacement qu'il peut générer lors de son immersion. Toutes les théories d'intervention sont soucieuses de mettre à la portée de tous, scientifiques comme acteurs du terrain, leurs concepts théoriques 
comme leurs résultats empiriques. Complémentaire à tous les autres courants sociologiques, n'en excluant aucun, la sociologie d'intervention se donne pour objectif de se «socialiser» (Hess, 198I), c'est-à-dire de proposer une lecture scientifique et sensible de la société qui soit accessible à tous et utilisable en situation. S’inspirant de la distinction faite par Chris Argyris entre connaissances applicables et connaissances utilisables, Crozier explique que les premières permettent de comprendre une situation et les comportements des individus. En revanche, les secondes sont réellement mises en pratique de façon quasi expérimentale. Elles aboutissent à l'élaboration d'outils et de plans d'actions immédiatement exploitables par les acteurs du terrain. Le problème, explique Crozier, vient de ce que «la majeure partie de la recherche en sciences sociales est applicable, mais pas utilisable» (2000, p. 292). La sociologie d'intervention tente de remédier à l'insuffisance de liens entre recherche et action. Pour autant, elle ne doit pas négliger chaque fois quelle le peut la montée en généralité. S'il est fréquent d’adresser aux sociologues du vécu le reproche d'une faiblesse théorique dans la mesure où ils n’en resteraient quaux «effets chauds » de l'expérience immédiate (Hanique, 2012, p. I25), ce reproche n’est pas justifié. Faire de la sociologie d'intervention n'induit pas de fait le renoncement à la conceptualisation. S’il s'agit pour le sociologue de «répondre à une question précise», il doit aussi chercher à «la déplacer pour la relier à des problèmes plus fondamentaux » (Trépos, 1996, p. 44). Sensible au principe le playsien de «médiation méthodique», l’analyse ne peut se limiter aux seules «caractéristiques de la situation immédiate, en s'isolant de l'ensemble des conditions générales, structurelles et historiques dans lesquelles s'établissent ces relations» (Schnapper, I999, p. I0). Les études microsociales de Gilles Herreros et de Bruno Milly (20II) sur les organisations hospitalières et médico-sociales, celles de Michel Crozier (2007), Dominique Felder (2007) et Marc Uhalde (200I) sur les entreprises industrielles et de service, celle de Denis Bernardeau Moreau (20I3) sur les organisations associatives et de loisirs, montrent qu'il est possible de dégager des tendances de fond sur l'évolution des sociétés. Pour autant, s'il est vrai que les éclairages locaux sont autant de miroirs reflétant les transformations sociétales à l'œuvre, il reste que toutes ces analyses se veulent être, avant tout, une source d'enrichissement pour les individus en situation qui attendent, du sociologue, une aide à l’amélioration de leur vie quotidienne. 


\section{BIBLIOGRAPHIE}

ALINSKY Saul, 1978, Manuel de l'animateur social, Paris, Seuil, «Point».

ARNAULT Françoise, 1984, «Frédéric Le Play, de la métallurgie à la science sociale», Revue française de sociologie, 25-3, p.437-457.

BEAUD Stéphane, PIALOUX Michel, 1999, Retour sur la condition ouvrière: enquête aux usines Peugeot de Sochaux-Montbéliard, Paris, Fayard.

BERNARDEAU MOREAU Denis, 2013, La Sociologie d'intervention. Le sociologue dans les organisations associatives, sportives et de loisirs, Paris, L'Harmattan, «Logiques sociales».

BERTHELOT Jean-Michel, 200I, Epistémologie des sciences sociales, Paris, PUF.

BOLTANSKI Luc, THEVENOT Laurent, 199I, De la justification. Les économies de la grandeur, Paris, Gallimard, «NRF essais ».

BOURDIEU Pierre (éd.), 1993, La Misère du monde, Paris, Seuil.

-, CHAMBOREDON Jean-Claude, PASSERON Jean-Claude, 1983, Le Métier de sociologue, New York Paris, Mouton éditeur, EHESS.

BURGESS Ernest, PARK Robert, 1921, Introduction to the science of sociology, Chicago, Illinois, University of Chicago Press.

CASTEL Robert, 2004, «La sociologie et la réponse à la demande sociale», in À quoi sert la sociologie?, Bernard Lahire (éd.), Paris, La Découverte, p. 66-67.

CHAPOULIE Jean-Michel, 1991, «La seconde fondation de la sociologie française, les États-Unis et la classe ouvrière», Revue Française de Sociologie, 32-3, p. 32I-364.

-, 2000, «Le travail de terrain, l'observation des actions et des interactions, et la sociologie», Sociétés Contemporaines, 40, p. 5-27.

CHAZEL François, 201I, «Comment lire Les Règles de la méthode sociologiques aujourd'hui?», in Durkheim fut-il durkheimien?, Raymond Boudon (éd.), Paris, Armand Colin, p. 27-40.

CHEYSSON Émile, 1896, Frédéric Le Play: l'homme, la méthode, la doctrine, Paris, Guillaumin. 
COUSIN Olivier, RUI Sandrine, 2010, L'Intervention sociologique. Histoire(s) et actualités d'une méthode, Rennes, PUR.

-, 20II, «La méthode de l'intervention sociologique. Évolutions et spécificités», Revue française de science politique, 61-3, p. 513-532.

CROZIER Michel, 195I, «Human Engineering», Les Temps Modernes, 69, p. 44-75.

-, 2000, À quoi sert la sociologie des organisations? Vers un nouveau raisonnement pour l'action, tome 2, Paris, Arslan.

-, 2007, Nouveau regard sur la société française, Paris, Odile Jacob.

-, FRIEDBERG Erhard, 1977, L'Acteur et le Système, Paris, Seuil.

DASSETTO Folice, 2006, «Sociologies et sociétés: une articulation à repenser?», Recherches sociologiques et anthropologiques, 37-I, p.7-26: <http://rsa.revues.org/604>.

DOLCI Danilo, 1957 (1956), Enquête à Palerme, Paris, Julliard, «Les Temps modernes».

DUBET François, 200I, «Plaidoyer pour l'intervention sociologique», in Vrancken et Kuty (200I), p. 89-1I0.

DU BOIS William Edward Burghardt, 1899, The Philadelphia Negro: A Social Study, Philadelphia, University of Pennsylvania Press.

DUBOST Jean, 1987, L'Intervention psycho-sociologique, Paris, PUF, «Sociologies».

-, 2006, Analyse sociale et sociologies d'intervention, Paris, L'Harmattan, «Histoire et mémoire de la formation».

DUMAS Bernard, SEGUIER Michel, 1999, Construire les actions collectives.

Développer les solidarités, Lyon, Chroniques sociales.

DUPUY François, 2004, Sociologie du changement. Pourquoi et comment changer les organisations, Paris, Dunod.

DURKHEIM Émile, 1999 (1895), Les Règles de la méthode sociologique, Paris, PUF, «Quadrige».

ENRIQUEZ Eugène, 2002, «Comptes rendus d'ouvrages», Revue internationale de Psychologie, VIII-19, p. 222-231.

FELDER Dominique, 2007, Sociologues dans l'action. La pratique professionnelle de l'intervention, Paris, L'Harmattan, «Questions sociologiques». 
FRIEDBERG Erhard, 1988 [1972], L'Analyse sociologique des organisations, Cahier 28, Pour. -, 1997 (1993), Le Pouvoir et la Règle. Dynamique de l'action organisée, Paris, Seuil, «Points-Essais».

FREIRE Paulo, 1996 (1964), L'Éducation, pratique de la liberté, ASDIC, Éditions W, «Décision locale».

GAULEJAC (de) Vincent, HANIQUE Fabienne, ROCHE Pierre (éds), 20I2, La Sociologie clinique. Enjeux théoriques et méthodologiques, Paris, Érès, «sociologie clinique».

GIDDENS Anthony, 1987 (1984), La Constitution de la société, Paris, PUF, Sociologies.

GILBERT Yves, 2009, Espace public et sociologie d'intervention, Perpignan, Presses universitaires de Perpignan.

-, BALDELLI Brigitte (éds), 20II, Intervention sociale et implication. Chercheurs, praticiens et usagers. Déconstruction, co-constructions et réciprocités, Perpignan, PUP, «Études».

HAMEL Jacques, 2007, « De la nature réflexive de la sociologie et de la disparition de son objet », Revue européenne des sciences sociales, XLV-139, p. 45-56.

HANIQUE Fabienne, 20I2, «De la sociologie compréhensive à la sociologie clinique», in Gaulejac (de), Hanique et Roche (2012), p. 105-130.

HERREROS Gilles, 200I, «Sociologie d'intervention: Pour une radicalisation de quelques principes», in Vrancken et Kuty (200I), p. 273-298.

-, 2008, Au-delà de la sociologie des organisations. Sciences sociales et intervention, Paris, Érès, Sociologie économique.

-, 2009, Pour une sociologie d'intervention, Toulouse, Érès.

HESS Rémi, 198I, La Sociologie d'intervention, Paris, PUF, «Le sociologue».

-, SAVOYE Antoine, 1993, L'Analyse institutionnelle, Paris, PUF, «Que sais-je?».

HESS Rémi, AUTHIER Michel, 1994, L’Analyse institutionnelle, Paris, PUF.

HUMBERT Colette, MERLO Jean, 1978, L'Enquête conscientisante. Problèmes et méthodes, L'Harmattan, Inodep-Document de travail.

KALOARA Bernard, SAVOYE Antoine, 1989, Les Inventeurs oubliés. Le Play et ses continuateurs aux origines des sciences sociales, Seyssel, Champ Vallon, «Milieux». 
LAPASSADE George, 1996, Les Microsociologies, Paris, Anthropos.

-, (1965) 2006, Groupes Organisations Institutions, Paris, Economica, «Anthropos».

LAPEYRONNIE Didier, 2004, «L'académisme radical ou le monologue sociologique.

Avec qui parlent les sociologues? », Revue française de sociologie, 45-4, p. 62I-65I.

LEGRAND Monique, VRANCKEN Didier, 2004, L'Expertise du sociologue, Paris, L'Harmattan, «Logiques Sociales».

LENOIR Rémi, CHAMPAGNE Patrick, MERLLIÉ Dominique, PINTO Louis, 1989, Initiation à la pratique sociologique, Paris, Dunod.

LE PLAY Frédéric, 1848, Descriptions des procédés métallurgiques employés dans le pays de Galles pour la fabrication du cuivre, et recherches sur l'état actuel et sur l'avenir probable de la production et du commerce de ce métal, Paris, Thunot et Cie.

-, I88I, La Constitution essentielle de l'humanité. Exposé des principes et des coutumes qui créent la prospérité ou la souffrance des nations, Tours, Marne.

-, 2000 (I862), «Instruction sur la méthode d'observation dite des monographies de familles propres à l'ouvrage intitulé Les Ouvriers européens», Les Études sociales, 131-132 p. 203-220.

MARTUCELLI Danilo, 199I, Histoire, intervention sociologique et mouvements sociaux, Séminaire CADIS, Roméotypé, Arcachon.

MAUSS Marcel, 1968, Sociologie et anthropologie, Paris, PUF.

MINGUET Guy, 200I, «Taxinomie de modèles sociologiques d'intervention», in Vrancken et Kuty (200I), p. 19-68.

MILLY Bruno, HERREROS Gilles, 20II, La Qualité à l'hôpital. Un regard sociologique, Paris, L'Harmattan, «Conception et Dynamique des Organisations».

MCCLUNG LEE Alfred, 1966, Multi-valent Man, New York, George Braziller.

MORIN Edgar, 1970, La Rumeur d'Orléans, Paris, Seuil, «L'histoire immédiate».

OSTY Florence, 200I, «Sociologie de l'entreprise et pratiques d'intervention in Vrancken et Kuty (200I), p. I67-190.

PAUGAM Serge, 2008, La Pratique de la sociologie, Paris, PUF. 
PICHAULT François, 2006, «L'intervention en organisation: Une question de légitimation systémique», Revue internationale de Psychosociologie, XII-26, p. 63-96.

PINÇON Michel, PINÇON-CHARLOT Monique, 2007, Les Ghettos du gotha. Comment la bourgeoisie défend ses espaces, Paris, Seuil.

PIRIOU Odile, 2006, La Face cachée de la sociologie. À la découverte des sociologues praticiens, Paris, Belin, Perspectives sociologiques.

RAVON Bertrand, 2009, «Vers une clinique du social?», in La Souffrance sociale.

Nouveau malaise de la civilisation, Marc-Henry Soulet (éd.), Académic Press Fribourg, p. $153-162$.

RORTY Richard, 1994, Objectivisme, relativisme et vérité, Paris, PUF, «L'interrogation philosophique».

SAVOYE Antoine, 1979, Genèse de la sociologie d'intervention, Thèse de doctorat de troisième cycle de sociologie, Université Paris X (fonds documentaire du CEDIAS).

-, 1981, «Les continuateurs de Le Play au tournant du siècle», Revue Française de Sociologie, 2-3, p. 315-344.

-, 1994, Les Débuts de la sociologie empirique, Paris, Meridien Klincksieck.

-, AUDREN Frédéric, 2008, Naissance de l'ingénieur social: Les ingénieurs des mines et la science sociale au XIXe siècle, Paris, Presses de l'école des Mines, «Sciences sociales».

SCHNAPPER Dominique, 1999, La Compréhension sociologique: démarche de l'analyse typologique, Paris, PUF.

-, 2010, Une sociologue au Conseil Constitutionnel, Paris, Gallimard, NRF Essais.

-, 20। I, «L'expérience-enquête au Conseil constitutionnel», Sociologie, 2-3: $<$ http://sociologie.revues.org//035>.

SIMON Pierre-Jean, 1991, Histoire de la sociologie, Paris, PUF, «Fondamental».

SMALL Albion, VINCENT George, 1894, An introduction to the study of society, New York, Cincinnati, American Book Company.

TOURAINE Alain, 1978, La Voix et le Regard: sociologie des mouvements sociaux, Paris, Seuil.

-, 1984, Le Retour de l’acteur, Paris, Fayard, «Essai de sociologie». 
TOURVILLE Henri (de), 1886, «La science sociale est-elle une science ?», in La Science sociale, janvier p.9-21, février p.97-109, avril p289-304, décembre p.493-516.

TREPOS Jean-Yves, 1996, La Sociologie de l'expertise, Paris, PUF, «Que sais-je?».

UHALDE Marc (éd.), 200I, L'Intervention sociologique en entreprise. De la crise à la régulation sociale, Paris, Desclée de Brouwer, «Sociologie économique».

VRANCKEN Didier, 1995, «Les tensions du métier de sociologue entre fondamentalisme et approche clinicienne», in La Sociologie et ses métiers, Monique Legrand, Jean-François Guillaume et Didier Vrancken (éds), Paris, L'Harmattan, «Logiques sociales», p. 35I-358.

-, 200 I, « L'intervention au cœur de la sociologie», in Vrancken et Kuty (200 I), p. $311-322$.

-, KUTY Olgierd, 200I, La Sociologie et I'Intervention. Enjeux et perspectives, Paris, De Boeck Université, «Ouvertures sociologiques».

WACQUANT Loïc, 200I, Corps et âme. Carnets ethnographiques d'un apprenti boxeur, Marseille et Montréal, Agone, Comeau et Nadeau.

WEBER Florence, 200I, Le travail à côté. Etude d'ethnographie ouvrière, Paris, Éditions de l'EHESS.

WORMS René, 1918 (1904), Philosophie des sciences sociales, II. Méthode des sciences sociales, Paris, Giard et Brière. 